\title{
Design of a Framework for Data-Intensive Wide-Area Applications *
}

\author{
Michael D. Beynon ${ }^{\dagger}$, Tahsin $\operatorname{Kurc}^{\dagger}$, Alan Sussman ${ }^{\dagger}$, Joel Saltz ${ }^{\dagger+}$ \\ $\dagger$ Institute for Advanced \\ Computer Studies \\ and \\ Dept. of Computer Science \\ University of Maryland \\ + Dept. of Pathology \\ Johns Hopkins Medical Institutions \\ Baltimore, MD 21287 \\ College Park, MD 20742 \\ $\{$ beynon, kurc, als, saltz\}ecs.umd.edu
}

\begin{abstract}
Applications that use collections of very large, distributed datasets have become an increasingly important part of science and engineering. With high performance wide-area networks becoming more pervasive, there is interest in making collective use of distributed computational and data resources. Recent work has converged to the notion of the Grid, which attempts to uniformly present a heterogeneous collection of distributed resources. Current Grid research covers many areas from low level infrastructure issues to high level application concerns. However, providing support for efficient exploration and processing of very large scientific datasets stored in distributed archival storage systems remains a challenging research issue.

We have initiated an effort that focuses on developing efficient data-intensive applications in a Grid environment. In this paper, we present a framework, called filter-stream programming, that represents the processing units of a dataintensive application as a set of filters, which are designed to be efficient in their use of memory and scratch space. We describe a prototype infrastructure that supports execution of applications using the proposed framework. We present the implementation of two applications using the filter-stream programming framework, and discuss experimental results demonstrating the effects of heterogeneous resources on application performance.
\end{abstract}

${ }^{*}$ This research was supported by the National Science Foundation under Grants \#ASC-9619020 (UC Subcontract\#10152408), and by the Office of Naval Research under Grant \#N66001-97-C-8534.

\section{Introduction}

Increasingly powerful computers have made it possible for computational scientists and engineers to model physical phenomena in greater detail. As a result, overwhelming amounts of experimental data are being generated by scientific and engineering simulations. In addition, large amounts of data are being gathered by sensors of various sorts, attached to devices such as satellites and microscopes. There are many examples of large useful datasets from simulations [26, 29, 33], sensor data [25, 28], and medical imaging [2] (pathology, MRI, CT scan, etc.). The primary goal of generating data through large scale simulations or sensors is to better understand the causes and effects of physical phenomena. Understanding is achieved through running analysis codes on the stored data, or by a more interactive visualization that relies on the ability to gain insight from looking at a complex system. Thus, both data analysis and visual exploration of large datasets plays an increasingly important role in many domains of scientific research. Decision support database applications are similar to scientific applications because they deal with large quantities of data (relational data), and need to perform significant computation in processing the data. The value provided by decision support systems and data-mining algorithms depend greatly on the amount of data, and hence businesses are inclined to retain as much data as possible.

Disks continue to become larger and cheaper making them commodity items. This helps to make it relatively easy to setup a large set of archival storage disks at a relatively low cost. For example, to build a large disk farm out of commodity PC components for the lowest current price: $\$ 400$ for a motherboard with a Celeron or AMD K6-2 400MHz cpu and 64MB memory [9], four 40GB EIDE disks at \$254 each [10] and a fast ethernet interconnect (100 Mbps), a 
farm of 8 PCs can present 1.25TB of disk space for less than $\$ 15 \mathrm{~K}$. The price point is sufficiently low to enable many such disk collections to be setup independently at multiple disparate locations, where local storage needs dictate. We anticipate that this trend will result in the emergence of $i s$ lands of data, where cheap archival storage systems will be used to hold large locally generated datasets. Use of computation farms also is important for handling very large datasets in a reasonable amount of time. Oftentimes, high performance computation farms are where the data is generated (as in large scientific simulations), and the data may reside locally on the computation farm in an archival storage system such as HPSS [22]. Thanks to high-performance networks, increasing numbers of computation farms have become accessible across a wide-area network. These computation farms span a spectrum of widely varying configurations and computation power, from relatively inexpensive network of workstations and PC clusters to very expensive high-performance machines, providing computing performance in the order of Teraflops.

These trends combine to present a new opportunity: very large distributed datasets that can be used by applications for computationally and data intensive analysis, exploration and visualization.

Consider the following scenario: A scientist wants to compare properties of a $3 \mathrm{D}$ reconstructed view of a raw dataset recently generated at a collaborating institution, with the properties of a large collection of reference datasets. The 3D reconstruction operation involves retrieving portions of 2D slices from the regions in question, and then performing feature recognition and interpolating between the slices to extract the important 3D features. A description of these features and the associated properties are then compared against a database of known features, and some appropriate similarity measure is computed. The final result is the set of reference features found that are close in some way to those found in the new raw dataset, along with the corresponding view renderings to visualize.

Consider the problems that can occur when the application is executed in a Grid [16] environment. That is, the required resources (new raw dataset, reference database, and the scientist) are all at distributed locations in a wide-area network as seen in Figure 1. The reference database is likely to be stored in an image library, since the dataset is large and useful to many users. The new raw dataset is stored at the site where the sensor readings were taken. If the hosts containing the data are low-power archival systems that make the execution of the $3 \mathrm{D}$ reconstruction code prohibitively expensive, it becomes unclear how to structure the application for efficient execution. Ideally we would like to execute portions of the application at strategic points in the collection of machines. A set of possible locations for performing computation is indicated in the figure by question marks.

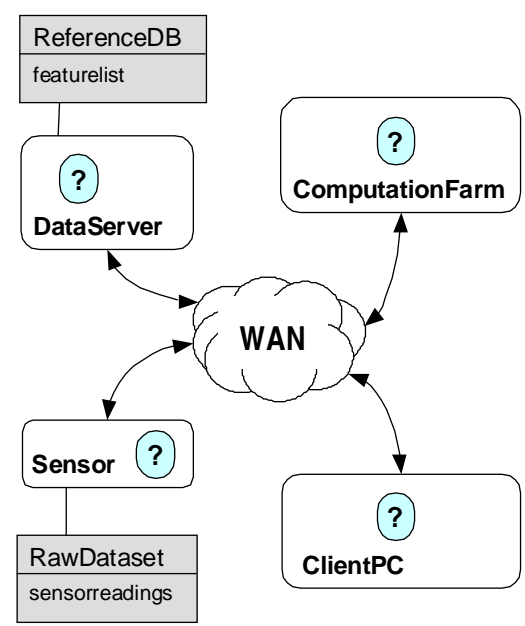

\section{Figure 1. 3D reconstruction/visualization sce- nario on distributed collection of resources.}

For example, if the portion of code that performs the range select on the new raw dataset could be run on the host where the data lives, the amount of data to be transmitted over the wide-area network (WAN) would be reduced. The computation farm is an ideal location for the feature recognition and $3 \mathrm{D}$ reconstruction due to the parallelism inherent in the codes. Given the set of features that were identified, it would be efficient to perform the selection of similar features from the reference database on the data server where the database is located. The low end PC where the scientist is located can be used to collect the 3D rendering and the similar feature information for interactive presentation to the scientist.

The success of this scenario depends on the application allowing portions of its computation to be executed in a distributed fashion. Beyond the mere possibility of execution in a distributed environment is the question of how efficient the application is. One interpretation of efficiency in this context is the ratio of useful data transmitted to the total amount of data transmitted between any two pieces of the application. For example, if an application transmitted a full dataset from a remote host, and discarded a large portion not required by subsequent processing, then this would not be considered efficient operation.

We have initiated an effort to investigate and develop methodologies and a framework for efficient execution of applications that make use of distributed collections of datasets in a Grid environment. There are two main challenges in developing efficient applications in a Grid environment:

- The Grid is composed of collections of heterogeneous resources. The characteristics, capacity and power of resources, including storage, computation, and network, vary widely. This requires that applications 
should be structured to accommodate the heterogeneous nature of the Grid.

- These distributed resources can be shared by many applications. This requires that applications should be designed to be optimized in their use of shared resources.

In order to address these challenges, we are investigating:

- Methodologies and a framework for structuring applications. In particular, we address decomposition of application processing into components and placement of these components onto a collection of heterogeneous resources that will aid efficient execution.

- Feasibility and effects of exposing application structure and characteristics. In particular, we address exposing resource requirements and the communication pattern between application components, and how this extra application structure information can be used.

- An infrastructure for providing execution of applications that conform to the developed framework.

In this paper, we present a framework, called filterstream programming, that represents the processing in a data-intensive application as a set of processing units, referred to here as filters, which are designed to be efficient in their use of memory and scratch space. In this framework, data exchange between any two filters is described via streams, which are uni-directional pipes that deliver data in fixed size buffers. We describe a prototype infrastructure that provides support for execution of applications using the proposed framework. We present the implementation of two applications in filter-stream programming framework, and experimental results to demonstrate the effects of heterogeneous resources on the performance of the applications.

\section{The Proposed Approach}

In this section, we present a framework, called the filterstream programming model. The basic ideas are to (1) constrain application components to allow for location independence, which is necessary for execution in a distributed environment, and (2) expose the application communication pattern and resource requirements, allowing a runtime system to aid in efficient execution. We should note that any programming model (e.g., message passing) modified to expose similar constraints could be employed in place of the filterstream programming model we describe.

The programming model used in this work is derived from the stream-based programming model, originally developed for Active Disks [1,35]. Many stream-based algorithms were developed and analyzed for Active Disks. These algorithms carry out a variety of data transformations that arise in earth science applications and applications of standard relational database sort, select and join operations. In this work we extend these algorithms and investigate the application of filters and the stream-based programming model in a Grid environment.

In the filter-stream programming model, an application is represented by a collection of filters. A filter is a portion of the full application that performs some amount of work. Filters are required to pre-disclose dynamic memory and scratch space needs. Communication with other filters is solely through the use of streams. A stream is a communication abstraction that allows fixed sized untyped data buffers to be transported from one filter to another. An example set of filters for the motivating example is shown in Figure 2. A simple example of this model is Unix system pipes, where the standard output of a process is used as standard input for another process. Unix pipes represent a linear chain of filters, each of which have a single input stream and a single output stream. The filter-stream model allows for arbitrary graphs of filters with any number of input and output streams.

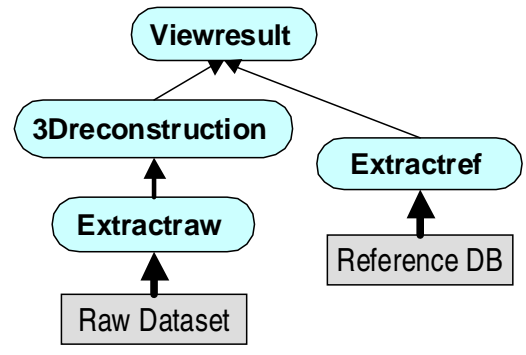

\section{Figure 2. 3D reconstruction application de- composed into filters.}

The process of manually restructuring an application using this model is referred to as decomposing the application. In choosing the appropriate decomposition, we need to consider the complete data flow path from data generation to ultimate consumption and the target machine configuration, which can be a distributed collection of heterogeneous machines. The main goal is to achieve efficient use of limited resources in a distributed and heterogeneous environment. The choice of decomposition can have a significant impact on efficiency and performance. Too many filters could mean there is not much work for individual filters, which would cause the system to spend much of its time moving data around and little time performing useful work on the data. Too few filters could limit the ability of the overall system to execute filters concurrently. Similarly, sending data over streams in very small pieces can make the overhead of the runtime system too large. If possible, an ideal granularity size should balance the amount of computation and communication such that the overall processing time across all fil- 
ters does not exhibit a penalty merely because the computation is distributed.

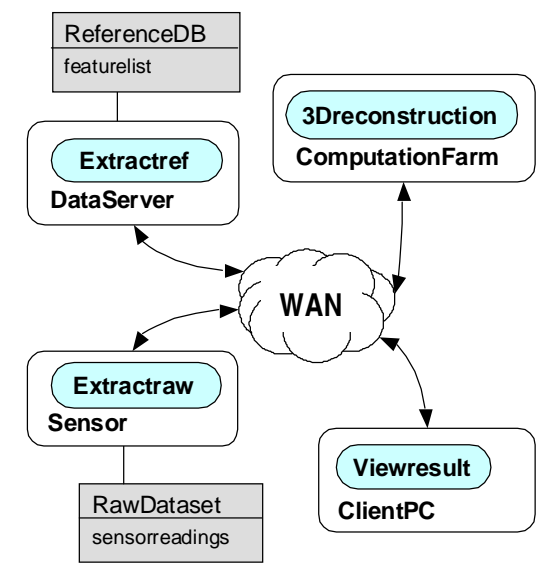

\section{Figure 3. Possible placement of 3D recon- struction application filters.}

Given a set of filters, the runtime mapping of filters onto various hosts in a wide-area grid environment is referred to as placement. Figure 3 shows a possible placement of the filters described for the motivating scenario. The choice of placement represents the main degree of freedom in affecting application performance by:

- placing filters with affinity to data sources near the sources,

- minimizing communication volume on slow links,

- co-locating filters with large communication volume,

- placing computationally intensive filters on less loaded hosts,

- pipelining application filters by concurrent execution.

Note that a placement decision is not assumed to be static, and the programming model explicitly supports the notion of stopping a set of filters and replacing them with possibly a new set of filters with a different placement.

A runtime system infrastructure is used to support the execution of applications that are structured in the filter-stream programming model. In the following sections we present a prototype infrastructure for executing application filters, and present implementations of an image processing application and a database application using the filter-stream programming model.

\section{Related Work}

There is a large body of hardware and software research on archival storage systems, including distributed parallel storage systems [24], file systems [34], image servers [32], and data warehouses [23]. Several research projects have focused on digital libraries and geographic information systems $[4,20]$ that access collections of archival storage systems, high-performance $\mathrm{I} / \mathrm{O}$ systems [8], tertiary storage systems [22] and remote I/O [19, 31]. Distributed storage systems attempt to provide large amounts of data to distributed clients. They present a uniform view of distributed data to applications, and transparently handle replicas and caching. This does not push the computation to the data as in our work, rather the data is migrated to the computation, but can achieve a similar result with an effective replacement policy and a warm cache. Another issue is finding the required data. The Storage Resource Broker (SRB) [31] provides uniform UNIX-like I/O interfaces and meta-data management services to locate and access collections of distributed data resources.

Distributed computing covers research that addresses ways to deal with distributed execution of application code in many different ways. Current work related to Grid computing $[7,14,16]$ attempts to provide a uniform view into a collection of distributed computational, network and storage resources, and to provide services for unified, secure, efficient and reliable access. However, providing support for efficient exploration and processing of very large scientific datasets stored in archival storage systems at distributed locations remains a challenging research issue, and the necessity of infrastructure to provide such support was recognized in recent Grid forums [21]. This support of processing and retrieval for efficient operation is exactly what our work is attempting to provide.

There is a large body of classic work on dataflow systems. The macro dataflow model $[30,36]$ describes an application as a sets of tasks, communication edges, edge communication costs and task computation costs. PYRROS [37] uses this model of application behavior and manual annotations to cluster, map, and schedule computation to nodes of a homogeneous parallel machine. As we target a heterogeneous grid environment, we expand on assumptions such as constant computation regardless of placement, which makes sense in a tightly coupled environment. There is also task parallel work in systems such as STRAND [12], PCN, Fortran M [13], and HPF [18], which are related due to the dataflow model and/or task parallelism used. Our work is different in that we are considering remote datasource affinity as a primary reason for decomposition, rather than an attempt to extract paralellism.

\section{A Prototype Infrastructure}

In this section, we describe a prototype infrastructure implementation that provides support for execution of applications developed using the filter-stream framework. This work is part of the DataCutter project [6], that provides 
services for subsetting and processing multi-dimensional datasets stored on archival storage systems.

\subsection{Filters}

A filter is specified by the code to execute, and a description of the input and output streams it will use. Currently, filter code is expressed using a $\mathrm{C}++$ language binding by sub-classing a provided filter base class. This base class provides a well-defined interface between the filter code and the system filter service. The description of input and output streams is specified in a separate configuration file (Figure 4).

Filters are constrained in several respects. First, undisclosed dynamic allocation of memory and local disk space is not allowed. Instead, the filter must pre-disclose and be granted scratch memory and disk space by the runtime system. The granted scratch space is allocated on behalf of the filter by the runtime system when the filter is instantiated. Later, the filter may make use of the granted scratch space as needed. One of the potential benefits of exposing resource requirements in this way is that runtime system can achieve a better placement of filters. For example, a filter can be run on a machine with enough memory to avoid paging, and two filters requesting large scratch space can be placed on two different machines. In addition, the runtime system can potentially perform better scheduling of co-located filters on a machine. One of our goals in this project is to investigate and assess the potential benefits of pre-allocating memory, when it will really be important, and implications for structuring applications. In order to accomplish this, we plan to compare standard versions of target applications with filterstream based implementations in subsequent work.

The interface for filters consists of an initialization function, a processing function, and a finalization function:

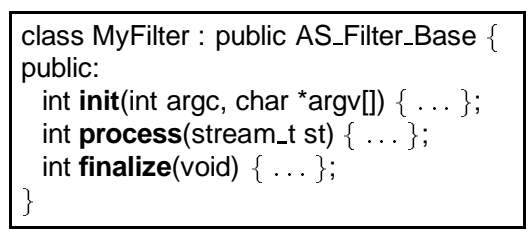

The init function is called when the filter is instantiated, and is passed parameters with the command line arguments used when the application was started. This is where a filter would request scratch memory space for use during later processing, for example. The process function is called to handle data arriving on the input streams in buffers from the sending filter. The parameter passed to the process function contains arrays of descriptors for the sets of input streams and output streams this filter can use. The filter can only read and write from/to the provided streams. No new streams can be created by the filter at runtime. The finalize function is called after all processing is finished and the filter is ready to terminate. This is where a filter would release any resources in use.

Another restriction is that a filter cannot change the source of its input streams nor the sinks of its output streams. This has two advantages. First, a filter does not need to handle buffering and scheduling for its own communication, thereby reducing the complexity of filters. Second, the location of filters is transparent, allowing filters to be placed at different locations initially and relocated as system resource constraints change.

Filters are the unit of placement. Each filter can potentially be executed on a different host. In addition, a filter's location may change at discrete application-defined intervals during the course of execution. Note this does not imply true migration of code and state, but rather placement can be recomputed and the filter can be stopped on the original host and a new copy re-instantiated on the new host. There is a limited mechanism for a final state transfer by a single buffer transfer from the old instance to the new instance. This approach avoids many of the details involved in checkpointing and process migration [11], while retaining most of the benefits. Filters need to be structured appropriately to handle such events. For cases when this is not desirable, a filter can be pinned to a particular host, which means the filter will always be placed on that host. This host affinity is useful for some situations, such as when runtime libraries or auxiliary data files only exist on a particular host, but does limit placement flexibility.

\subsection{Streams}

A stream is an abstraction used for filter communication. Since the placement of filters is largely unknown until runtime, this mechanism is used to achieve locationindependent filter code because stream names are used rather than endpoint location on a specific host. A stream is used to specify how filters are logically connected, and to provide the glue at runtime to attach an input stream for one filter to an output stream of another. All transfers to and from streams are through a provided buffer abstraction. A buffer represents a contiguous memory region containing useful data. The buffer contains a pointer to the start, the length of the portion containing useful data, and the maximum size of the buffer. In the current prototype implementation we are using TCP for stream communication, but any point-to-point communication library could be added, such as Nexus [17].

The streams are specified in a global sense, separate from the application code. For each filter, a list of input and output streams is required. This discloses all potential filter communication pairs for the entire execution of the application. Given a set of filters with stream connectivity information, 
we can build a task graph where the nodes represent the filters, and the edges represent stream connections. For example, given three filters A, B and C, with data being sent from A to both B and C, and from B to C, the specification and resulting task graph are seen in Figure 4. Each filter in the spec appears in a section labeled [filter. $<$ name $>$ ]. For each section, two optional entries ins and outs can appear containing the list of input and output stream names respectively.

\begin{tabular}{|l|}
\hline [filter.A] \\
outs $=$ stream1 stream3 \\
[filter.B] \\
ins $=$ stream1 \\
outs $=$ stream2 \\
[filter.C] \\
ins = stream2 stream3
\end{tabular}

(a) filter/stream spec

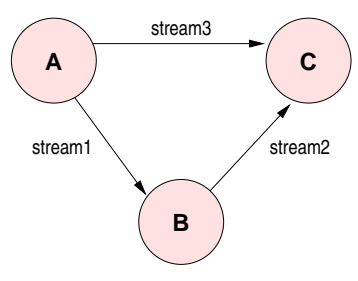

(b) resulting graph

\section{Figure 4. Sample filter/stream specification.}

In addition to the above inter-filter streams, we allow for two other types of streams: ${ }^{1}$ File Streams and External Communication Streams. Files Streams are used to read and write to files stored in local scratch disk space or local permanent disk storage. The file stream abstraction further insulates the filter code from specifics about the host system. This provides a measure of safety between co-located filters, since one filter cannot access another filter's scratch disk space. The permanent disk storage presents a uniform file system to all filters, similar to a traditional file system. Thus a filter with sufficient authorization can read files in permanent disk storage written by another filter. External Communication Streams are used to connect to, and receive connections from legacy or other non-filter application code.

\subsection{Execution Environment}

The execution service performs all the steps necessary to instantiate filters on particular machines, connect all the logical stream endpoints, and call the interface functions to allow filters to run.

The description of where to instantiate filters is provided by a placement specification. Currently, this is statically generated before the application is started. An example placement specification for the sample filters is:

$$
\begin{array}{|l}
\hline \text { [placement }] \\
\mathbf{A}=\text { host1.cs.umd.edu } \\
\mathbf{B}=\text { host2.cs.umd.edu } \\
\mathbf{C}=\text { host3.cs.umd.edu }
\end{array}
$$

\footnotetext{
${ }^{1}$ These are not yet implemented in the prototype.
}

The [placement] section is expected to contain one entry for each filter. The value is simply the host to execute the filter on. In general, this host can be a parallel machine, which implies multiple instances of the filter are created, but the prototype implementation does not yet support parallel filters. Security concerns have made it difficult to start processes on remote machines in a uniform manner. To solve this problem in the current prototype, an Application Execution Daemons (appd) must be run on every host used to execute filters. In the future, we plan to use existing Globus [15] services for process creation and authentication, in which case the Application Execution Daemons would not be needed. In addition, a single provided Directory Daemon (dird), which is similar to an LDAP server, is used to record the contact information (host, port, pid) for each appd. The dird is the only process that runs on a welldefined host and port. All other ports are ephemeral, and registered with the dird to later be queried. Based on a given placement specification, the execution of a filter-based application requires contacting the appd process on each host. A lookup is performed to find contact information for each required appd. Currently, we require an application binary to exist on every host, which must contain at least the code for the filters that will execute on that host. The binary can contain code for all the filters, and those filters not intended to run on a given host will not be instantiated at runtime. Currently we manually compile/copy the binaries as needed, but convenience procedures to do this will be added in the future.

The application is started by running the application binary on some host. This will become the console process, which performs no application processing such as running filters. The console process queries the dird process to get the relevant appd contact information, and then sends an execute command to each appd. The appd executes the application binary on that host, which in turn contacts the console process and performs some initial handshaking to setup the stream abstractions. In the current prototype, one POSIX thread is created for each filter that runs on the host, and a new instance of the application filter object is created. The thread calls the init interface function passing the command line arguments that were used when the console process was started. Next, the thread calls the process function. When this returns, all open streams are closed and the finalize function is called. Any remaining filter resources are released before the thread stops.

The multiple threads allow for fairness across filters on a single host, since all threads are executed with the same priority by the underlying operating system. No one filter can starve another due to the time sharing semantics of POSIX threads. Of course the filters do need to be thread-safe with respect to each other. Based on the filter-stream programming model, this should be natural for most applications. 
Filters in this model are inherently isolated and communicate via system provided buffers, thus should be fairly easy to make thread-safe due to the lack of shared resources. One problem could be common library routines. For the cases where no thread-safe implementations exist, we provide filter level locks that can be used to wrap the offending calls. This is only an issue when thread safety problems exist between filters that run on the same host, thus in the same process. For the sample placement, filters A, B and C can all have thread safety violations, since they are all actually run in separate processes on three different hosts.

For cases when thread safety is a problem and lock wrapping will not work, the infrastructure could be augmented to optionally use a single thread for all filters on a given host. Control could use a dataflow model where scheduling is performed by the infrastructure for filters based on the arrival of input. Another alternative re-design is to make each filter execute as a separate process, thus avoiding all threading issues at the expense of increased filter communication costs on the same host. The use of a thread-per-filter-instance is a property of the current prototype implementation, and is not mandated by the overall model.

\subsection{Applicability}

Our approach is intended to be applicable to many common types of data-intensive applications that are emerging for use in a grid environment. The benefits of this approach result directly from two observations. The first is that the filter-stream framework exposes useful information, particularly application communication pattern and communication volume information. The second is that expressing the application processing as filters enables data volume reduction from remote data sources. These factors can be leveraged to improve application efficiency at runtime.

We recognize that the approach may not be effective for all application types, and are identifying characteristics that make applications ill-suited for this approach. Ill-suited in this case means performance will be no better than that of a generic message passing implementation, for example using MPI [27]. The first problem occurs when applications have high selectivity. This means nearly all the remote data is needed by the application, and no significant data reduction is achieved, which will nullify the benefits of application decomposition.

Applications that lack a clear task structure are also problematic. If the application cannot be divided cleanly into a set of filters, then placement choices are more limited for such a monolithic application. For example, if an application uses two remote data sources and cannot be divided into filters, we can execute the application at either data source (inputs), the client (output), or at an intermediate location. This will most likely be efficient only for data located at the execution site chosen, and inefficient for other input/output data sources/sinks.

The communication pattern and volume are significant characteristics that enable intelligent placement to overlap communication with computation and reduce high volume on slow network links. If the pattern or volume of communication is unknown, chaotic, very fine grained, or time varying, then it is difficult to perform an intelligent placement. For example, a communication pattern that involves all possible filters and is data dependent, where the destination for a piece of data is known only after its examination, will result in a conservative approximation of an all-to-all pattern with equal volume between all pairs of filters. There is no clear choice for placement in this case, because any possible good placement may only be known after execution has finished and the communication activity has been observed. Even worse, the observed communication pattern and volume may not be helpful for future runs, due to nondeterminism in such applications. Our approach assumes a single significant communication pattern and deterministic volume, which can be used for choosing placement for the entire execution. For the applications we are targeting, such as volume visualization, database decision support, and image processing, these assumptions appear to hold.

\section{Application: Image Processing}

The Virtual Microscope [2] is a query-response application that processes multi-dimensional image data to satisfy client queries. The dataset contains high power digitized images of microscope slides, which effectively forms a 3D dataset because each slide can contain multiple 2D focal planes at different depths. Images are stored at the highest magnification level, and the size of a single slide typically varies from $100 M B$ to $5 G B$, compressed. The system is required to provide interactive response times similar to a physical microscope, including continuously moving the stage and changing magnification. A typical query allows a client to request a $2 \mathrm{D}$ rectangular region at a particular magnification from within the bounds of a single focal plane. The processing for the query requires projecting high resolution data onto a grid of suitable resolution (governed by the desired magnification) and appropriately compositing pixels that map to a single grid point to avoid introducing spurious artifacts into the displayed image. The Virtual Microscope is useful for performing operations that are difficult with a physical microscope, such as simultaneous viewing and manipulation of a single slide by multiple users, or remote telepathology [2] where diagnosing pathologists are not required to be physically located near the slide. 


\subsection{Original Implementation}

The original Virtual Microscope system is composed of two components; a client to generate queries and display the results (i.e. images), and a server to process the queries. The server is composed of a frontend and a backend. The frontend interacts with clients; it receives queries from clients and forwards them to the backend. The backend consists of one or more processes, typically one per node of a parallel machine. The processing of a query is carried out entirely in the backend.

In order to achieve high I/O bandwidth, each focal plane in a slide is regularly partitioned into data chunks, each of which is a rectangular subregion of the $2 \mathrm{D}$ image. Data chunks are declustered across all backend local disks to achieve I/O parallelism. Each pixel in a chunk is associated with a coordinate (in $\mathrm{x}$ - and $\mathrm{y}$-dimensions) in the entire image. Each chunk has an associated minimum bounding rectangle (MBR) based on all the pixels in the chunk. An index is created using the MBR of each chunk. Since the image is regularly partitioned into rectangular regions, a simple computation can be used instead of a complex index search.

During query processing, the backend process finds the chunks that intersect the query region, and reads them from the local disks. Each data chunk is stored in compressed form (JPEG format), and must be first decompressed. Then, it is clipped to the query region. Afterwards, each clipped chunk is subsampled to achieve the zoom level (magnification) specified in the query. The resulting image blocks are directly sent to the client. The client viewer assembles and displays the image blocks from each of the backend processes to form the query output.

\subsection{Filter Implementation}

The filter decomposition used for the Virtual Microscope system [6] is shown in Figure 5. This filter pipeline structure is natural for query-response applications. The figure only depicts the main dataflow path of image data through the system; other low-volume streams related to the clientserver protocol are not shown for clarity. The thickness of the stream arrows indicate the relative volume of data that flows on the different streams.

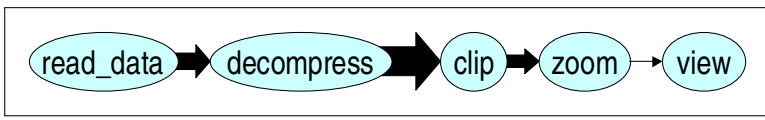

\section{Figure 5. Virtual Microscope decomposition}

In this implementation each of the main processing steps in the server is a filter:
- read_data: Full-resolution data chunks that intersect the query region are read from disk, and written to the output stream.

- decompress: Image blocks are read individually from the input stream. The block is decompressed using JPEG decompression and converted into a 3 byte RGB format. The image block is then written to the output stream.

- clip: Uncompressed image blocks are read from the input stream. Portions of the block that lie outside the query region are removed, and the clipped image block is written to the output stream.

- zoom: Image blocks are read from the input stream, subsampled to achieve the magnification requested in the query, and then written to the output stream.

- view: Image blocks are received for a given query, collected into a single reply, and sent to the client using the standard Virtual Microscope client/server protocol.

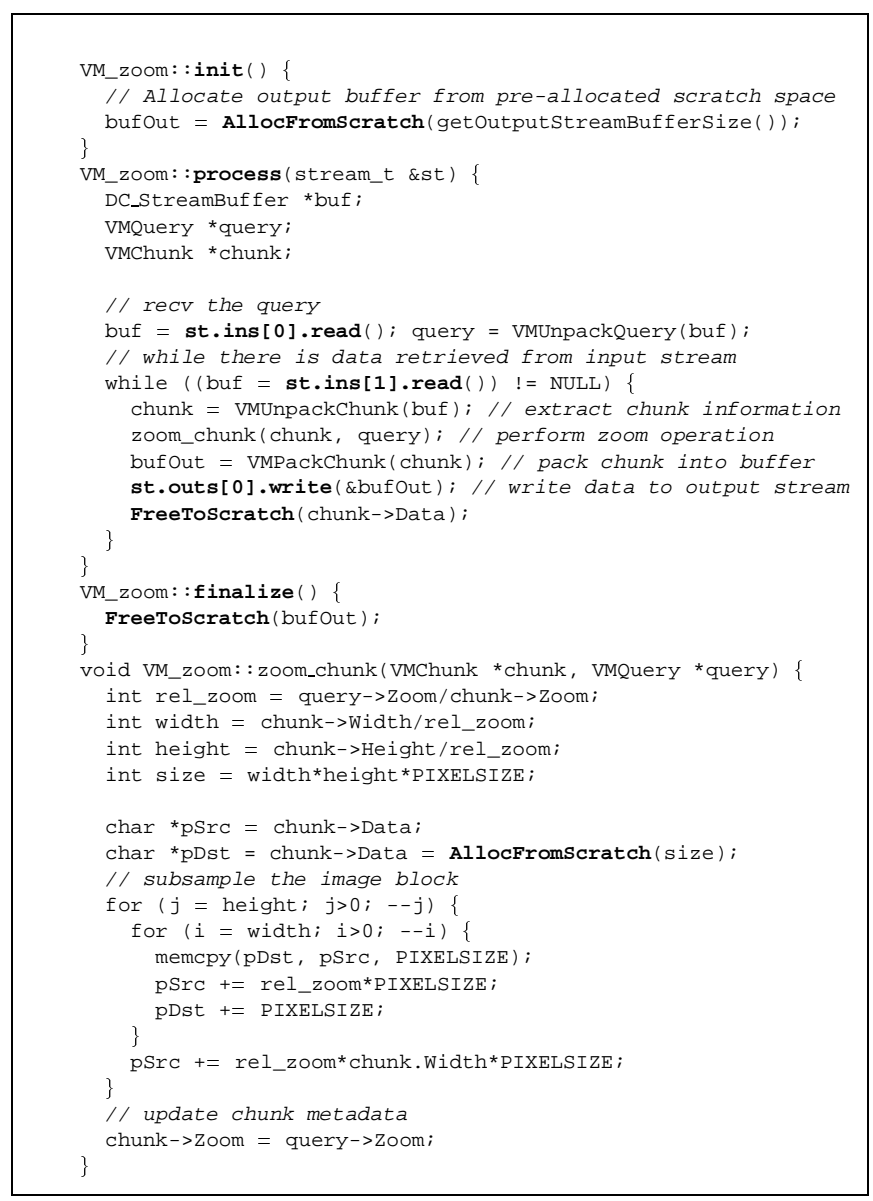

\section{Figure 6. The high-level code for zoom filter.}

Figure 6 illustrates the high-level code for the zoom filter, which has two input streams and one output stream. It reads the query from stream 0 (st.ins[0]) and data chunks 
from stream 1 (st.ins[1]), and subsamples the received data chunks using the zoom_chunk function. The zoom filter uses scratch space to store results during subsampling and to pack the subsampled chunk into the output buffer. The result is written to the output stream (st.outs[0]), which connects the filters zoom and view.

\subsection{Experimental Results}

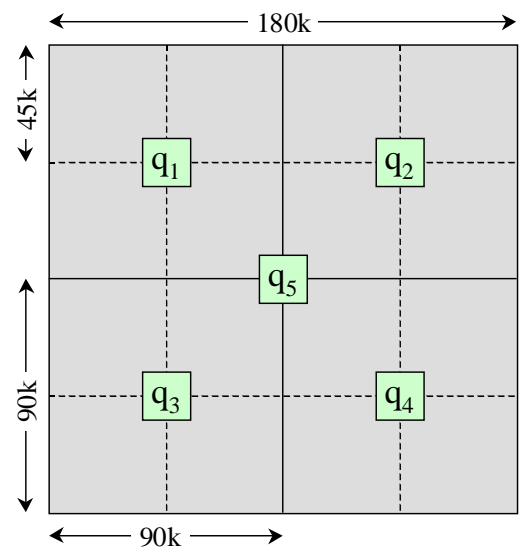

\begin{tabular}{|lrr|}
\hline Filter & Total Volume & Volume Per Chunk \\
\hline read data & $3.60 \mathrm{MB}$ & $102.52 \mathrm{~KB}$ \\
decompress & $83.42 \mathrm{MB}$ & $2373.04 \mathrm{~KB}$ \\
clip & $57.83 \mathrm{MB}$ & $1645.02 \mathrm{~KB}$ \\
zoom (no) & $57.83 \mathrm{MB}$ & $1645.02 \mathrm{~KB}$ \\
zoom (8) & $.90 \mathrm{MB}$ & $25.70 \mathrm{~KB}$ \\
\hline
\end{tabular}

Figure 7. 2D dataset and query regions. The table lists transmitted data sizes for $q_{5}$. zoom (no) is for no subsampling and zoom (8) is for a subsampling factor of 8 (in each of the two spatial dimensions).

Using the filters described in Section 5.2, we have implemented a simple data server for digitized microscopy images [6], stored in the IBM HPSS archival storage system at the University of Maryland. An existing Virtual Microscope client trace driver was used to drive the experiments. This driver was always executed on the same host as the view filter, which is referred to as the client host. The server host is where the read_data filter is run, which is the machine containing the disks with the dataset.

The HPSS setup has 10TB of tape storage space, 500GB of disk cache, and is accessed through a 10-node IBM SP. In all experiments we use a 4GB 2D compressed JPEG image dataset (90GB uncompressed), created by stitching together smaller digitized microscopy images. This dataset is equivalent to a digitized slide with a single focal plane that has $180 K \times 180 K$ RGB pixels. The $2 \mathrm{D}$ image is regularly partitioned into $200 \times 200$ data chunks and stored in HPSS in a set of files. We defined five possible queries, each of which covers $5 \times 5$ chunks of the image (see Figure 7). The execution times we will show are response times seen by the visualization client averaged over 5 repeated runs. For the presented experiments, we eliminated the effects of retrieving data stored on tape by insuring the data was staged to the HPSS disk cache before each run. We are using machines on our local area network for experimental repeatability, and will switch to hosts in a wide-area Grid environment once application behavior is sufficiently well-understood.

Overhead of Using Filters. The query execution times for the original optimized Virtual Microscope server versus the prototype filter implementation are shown in Figure 8. In this experiment the entire dataset is loaded from HPSS and stored on a single local disk attached to a SUN Ultra 1 workstation, because the original server can only access datasets stored on disks. The loading of the dataset took $4750 \mathrm{sec}-$ onds (1 hour 19 minutes). The original server is run as a single process, and all filters in the filter-stream implementation are executed on the same uniprocessor SUN workstation where the dataset has been pre-loaded. In both cases the client is run on another SUN Ultra 1 workstation connected to the local Ethernet segment. As is seen from the figure, the filter implementation does not introduce much overhead compared to the optimized original server. The percent increase in query execution time ranges from $6 \%$ to $30 \%$ across all queries. The filter version contains extra work not present in the original server, such as flattening of the chunk and metadata into a linear buffer on the sending filter, and expanding the chunk and metadata into the same structure in the receiving filter. This overhead is necessary when filters are located on distributed machines, but could be eliminated for the co-located case by instead sending a pointer to an inmemory structure, which would eliminate much of the overhead. This experiment is designed to be biased against the filter implementation to see what the overhead is in the decomposed version. We should also note that the timings do not include the time for loading the dataset from tape, which can substantially increase for larger datasets and datasets stored in archival storage systems across a wide-area network.

Varying the Processing. One node of the IBM SP is used to access the stored dataset, and the client was run on a SUN workstation connected to the SP node through the department Ethernet. We experimented with different placements of the filters by running some of the filters on the same SP node where the data is accessed, as well as on the SUN workstation where the client is run.

In Figure 9 we consider varying the placement of the filters under different processing requirements. Figures 9 (a) and (b) show the query execution times when the image is viewed at the highest magnification (no subsampling) and when the subsampling factor is 8 (i.e. every 8 th 


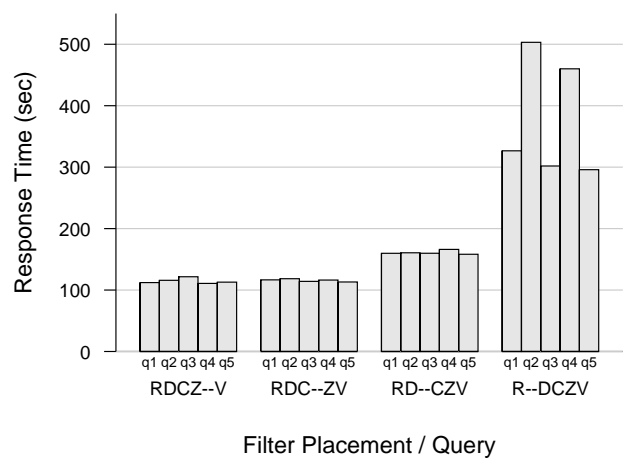

(a) no zooming (no subsampling)

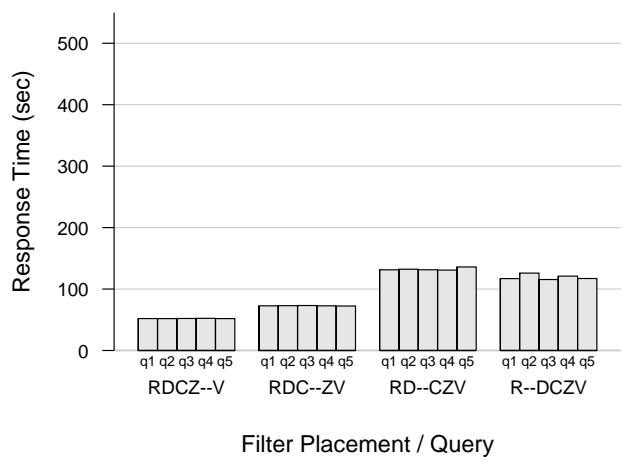

(b) subsampling by a factor of 8

Figure 9. Execution time of queries under varying processing (subsampling). R,D,C,Z,V denote the filters read_data, decompress, clip, zoom, and view, respectively. <server>-<client> denotes the placement of the filters in each set.

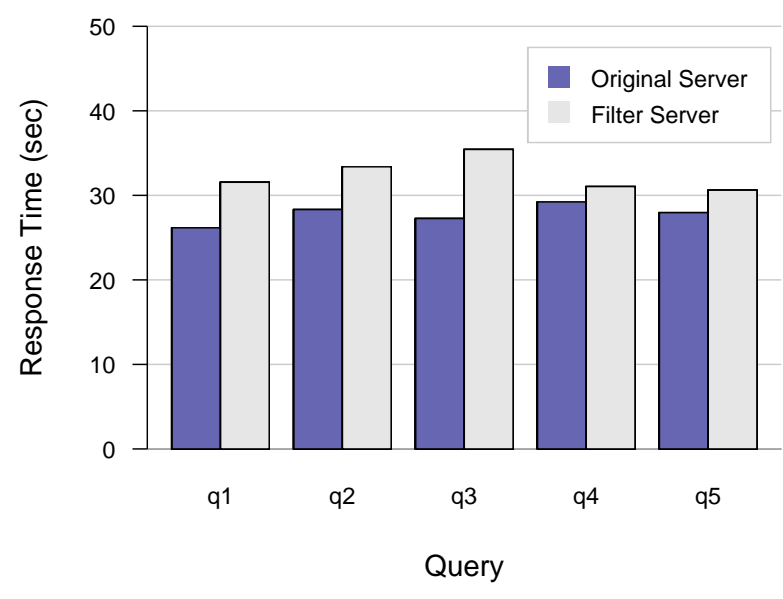

Figure 8. Query execution times for the original server and the filter implementation. (subsampling factor is 8 )

pixel in each dimension is output), respectively. There are three predominant factors in these experiments. The first is the placement of the most computationally intensive filter (decompress). The second is the volume of data transmitted between the two machines. The final factor is the amount of data sent by the view filter to the client driver. Consider the first two groups of bars in the figures. The difference between the groups within each figure is the placement of the zoom filter on the server (RDCZ-V) or client host
(RDC-ZV). When there is no subsampling, query execution times remain almost the same for both placements, because the volume of data transfer between the server and client is the same in both cases. In the case of subsampling, the placement of the zoom filter makes a difference, because the volume of data sent from the server to the client decreases if the zoom filter is executed at the server. Now consider the last two groups of bars in the figures. The difference between the groups within each figure is the placement of the decompress filter (RD-CZV or R-DCZV). For no subsampling case, the time increases substantially when decompress is placed on the client, because of the combined effects of the most computationally intensive filter (decompress) and the high amount of data being processed by view and sent to the client driver. When there is subsampling, the query execution time is not as high, because the amount of data processed by view and sent to the client driver is much lower. These experiments demonstrate the complex interactions between placement of computation and communication volume.

Varying the Server Load. In the next set of experiments (Figure 10), we consider varying the server load. We use the same experimental setup as for the previous experiment. In all experiments, we use a subsampling factor of 8 . Figures $10(a),(b)$, and (c) show query execution times when the server load is doubled, tripled, and quintupled, respectively. The different loads were emulated by artificially slowing down the set of filters running on the server host such that the total running time was delayed. For example, the zoom filter runs twice as long in the $2 \times$ case because the time is delayed. As server load increases (or the client host becomes rela- 


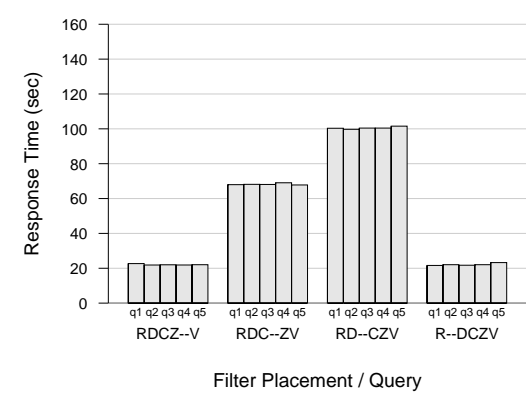

(a) 2x server load

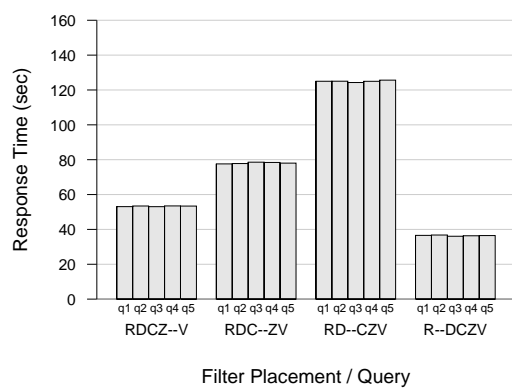

(b) $3 x$ server load

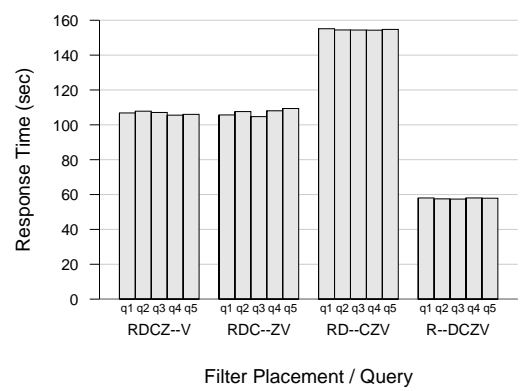

(c) $5 \mathrm{x}$ server load

Figure 10. Execution time of queries under varying server load. $2 \times$ means the server computation is delayed to double the execution time of a filter on the server, etc. (subsampling factor is 8 )

tively faster), running the filters on the client host achieves better performance. This result is not unexpected, but the experiment quantifies the effect for this particular configuration. The use of a different client to server network, or hosts with different relative speeds would significantly change the observed trends and trade-off points.

\section{Application: External Sort}

External sort has a long history of research in the database community and has resulted in many fast algorithms [3,5]. The application starts with a large unsorted data file that is partitioned across multiple nodes, and the output is a new partitioned data file that contains the same data sorted on a key field. The sample data file is based on a standard sorting benchmark that specifies 100 byte tuples, with the first 10 bytes being the sort key. The distribution of the key values is assumed to be uniform, both in terms of the unsorted file as a whole and for each partition. A recent record holder for the fastest external sort is NowSort [5], and we use the pipelined version of their two-pass parallel sort for our basic algorithm.

The algorithm proceeds in two phases. The first phase generates temporary sorted runs on each node, and the second phase produces the output sorted partition on each node. During the first phase, a reader reads chunks of tuples from the unsorted input file on disk, and partitions the records according to which node it will reside on when sorted, puts them into in-memory buffers, and when a buffer is full, sends it to the correct node. A writer collects tuples from all nodes, and when the in-memory buffer is full, sorts it using partial-radix sort ${ }^{2}$, and writes the sorted run to disk. This

\footnotetext{
${ }^{2}$ Making two passes over the keys with a radix size of 11-bits [3] plus a
}

first phase is over when all the unsorted input files have been processed, and written to disk as temporary sorted runs. For the second phase a merge-reader reads tuples from each local sorted run into merge input buffers. A merger selects the lowest-value key among all merge input buffers and copies it to an output buffer, from which the merge-writer appends buffers to the sorted output file on disk. This phase completes when tuples from all local runs have been merged.

\subsection{Filter Implementation}

The implementation of external sort using filters follows the above description. The location of the unsorted dataset dictates the number of nodes to be used for execution. There are two filters on each node, Partitioner and Sorter. The Partitioner filter reads buffers from the unsorted input file, and distributes the tuples into buckets based on the key value. When a bucket is full, it is sent over the stream that connects to the Sorter filter on the corresponding node. The Sorter continually reads buffers from the input streams, and extracts a portion of the key and appends it to a sort buffer. When the sort buffer becomes full, it is sorted and written to scratch space as a single temporary run. When all buffers have been read from the input streams, the merge phase begins with only the Sorter filters still executing. The Sorter filter then reads sorted tuples from each of the temporary run files and merges them into a single output buffer, and writes this buffer to the sorted output file on disk.

This application is essentially a parallel SPMD program, with an all-to-all communication pattern. This organization is in contrast to the Virtual Microscope application that was structured as a processing chain pipeline.

cleanup. 


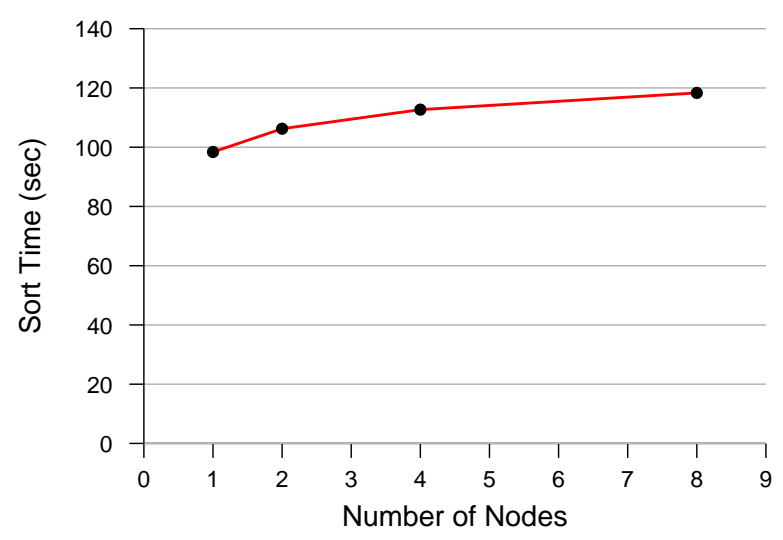

Figure 11. Sort execution time as number of nodes is increased. The dataset size is scaled with the number of nodes (128MB/node).

\subsection{Experimental Results}

The experimental setup is a 16 node cluster of dual $400 \mathrm{MHz}$ Pentium IIs with 256MB memory per node, running Linux kernel 2.2.12. There are two interconnects, a shared Ethernet segment, and a switched gigabit Ethernet channel. We use the faster switched interconnect for all experiments, and because of a problem with the network interface cards on some of the nodes, only use a maximum of 8 nodes in all experiments. The nodes are isolated from the rest of the network, and the cluster was not running other jobs during the experiments. Each node has a single Ultra2 SCSI disk. All data for a particular node, including temporary data, is stored on the single local disk. The dataset consists of a single 128MB unsorted file per node. The unsorted dataset was generated randomly with a uniform key distribution. The execution time for an experiment is the maximum time across all nodes used for the experiment. Each experiment is repeated for 5 trials, and the execution time shown represents the average of the trials. Both a Partitioner and a Sorter filter are executed on each node used in the experiments for Figures 11 and Figures 13(a)-(d), and two Partitioner and Sorter filters are executed on some of the nodes in the experiments for Figures 13(e) and (f). The disk cache was cleared between executions to insure a cold disk cache for each run. Note that we are using a tightly coupled cluster for experimental repeatability, and will be switching to hosts on a wide-area Grid environment when application behavior is better understood.

Scaling. The first experiment examines the scalability of the sort application as we increase the number of nodes and total dataset size. As seen in Figure 11, the application is

\begin{tabular}{|lrr|}
\hline Filter & Parameter & Full Memory \\
\hline $\begin{array}{l}\text { Partitioner } \\
\text { single disk buffer for reading tuples from the unsorted input file }\end{array}$ & 256 KB \\
\hline $\begin{array}{l}\text { Partitioner } \\
\text { shared space for all outgoing tuple buckets, before sending to Sorter filters }\end{array}$ & bucket_size & $1 \mathrm{MB}$ \\
\hline $\begin{array}{l}\text { Sorter (phase 1) } \\
\text { single buffer for storing extracted key and tuple pointer, before sorting and writ- } \\
\text { ing the temporary run }\end{array}$ & keybuf_size \\
\hline $\begin{array}{l}\text { Sorter (phase 2) } \\
\text { shared disk buffer for reading from all temporary runs during merge }\end{array}$ & $768 \mathrm{~KB}$ \\
\hline $\begin{array}{l}\text { Sorter (phase 2) } \\
\text { single disk buffer for writing sorted tuples to output file }\end{array}$ & Outputbuf \\
\hline
\end{tabular}

Figure 12. Memory parameters used by the sort filters. The Full Memory column contains the initial value for each parameter.

well-behaved. There is good scaling due to the fast interconnect not becoming saturated by the traffic generated by sort. This experiment demonstrates there is nothing inherent in the filter-stream based implementation that would otherwise limit its scalability.

Varying Memory Size. In this set of experiments we vary the amount of memory available for filters on some of the nodes while keeping it constant for filters on the remaining nodes. Our goal is to create a heterogeneous configuration in a controlled way, and observe the effects of heterogeneity on the application performance.

Figure 13 shows the execution times under varying memory constraints. The solid line in all of the graphs denotes the base case, in which the size of the memory is reduced equally across all nodes, and shows the change in the execution time. The amount of the Full memory case is determined empirically to minimize execution time while consuming the least memory (see Figure 12). Memory parameters are varied by halving the full memory amount for the $1 / 2$ case, and halving again for the $1 / 4$ case, etc. Constraining memory causes the filters to read/process/write data in smaller pieces, thus performance should suffer. As is shown by the solid line in the figure, the execution time increases as the size of the memory is decreased. In the experiments with heterogeneous memory configuration, we divide the eight nodes into two sets of four nodes. The first set of nodes retains the initial amount of memory (i.e., Full memory) for all runs, while the second set has their memory reduced for each case. The left bars for each case in each graph shows the maximum of the execution times on the nodes with full memory. Similarly, the right bar for each case in each graph shows the maximum of the execution times on the nodes with reduced memory. As is shown in Figure 13(a), we observe performance degradation similar to the base case. The nodes that use a constant amount of memory finish sooner, 
but the entire job runs no faster. In this experiment, both the input data to the Partitioner filter and the output of the Partitioner (i.e. the input data to the Sorter filter) on each node are regularly partitioned across all the nodes.

Notice that the total amount of memory across all nodes for this experiment is larger than that for the base case because half the nodes keep full memory. For example, for the $1 / 8$ memory case, $350 \%$ more memory was being used in aggregate than for the $1 / 8$ base case. Instead of a reduction in sort time, the extra memory results in a load imbalance between the two sets of four nodes. Hence, in the next experiment we partitioned the amount of input data for each node irregularly, to attempt to reduce overall execution time. Figure 13(b) shows that the execution time increases when we partition the input data based on available node memory, i.e., full nodes have more input data than nodes with reduced memory. This results from an increase in the time for the partitioning phase, because the Partitioner filters on the set of nodes with full memory have more input records to process. The execution time for the merge phase is effectively unchanged, because the amount of data sent to each node is unchanged. Figure 13(c) shows the result of partitioning the output of the Partitioner filter (and thus the merge phase work) according to the memory usage of the receiving node. This experiment, however, moves too much work to the nodes with full memory, so that those nodes become the longest running node set. To improve performance further, we followed two different approaches. In Figure 13(d), the Partitioner filter output is adjusted to balance the performance of both sets of nodes (approximately a 10\% reduction in the number of tuples assigned to a node for each $1 / 2$ reduction in memory usage). In this case, we observe better performance than the previous cases. In the second approach, we partitioned both the input data and the output of the Partitioner filter as was done in the experiment for Figure 13(c), but executed two Sorter and two Partitioner filters on the nodes with Full memory to take advantage of the dual processors available in each node. As is seen in Figure 13(e), the performance is better than for the previous cases. Finally, Figure 13(f) shows the combined effect of running two sets of filters on the nodes with full memory, and adjusting the Partitioner output to balance the workload across both set of nodes. As expected, this configuration performs better than all other cases. These experimental results clearly show that application-level workload handling and careful placement of filters can deal with heterogeneity, which can have a significant impact on performance. Questions that require further investigation include (1) "can we develop cost models for filters and for the application performance so that the placement of filters and workload handling can be done by the runtime system, with little intervention from the user?" and (2) "can we make use of exposing resource requirements and communication characteris- tics to develop accurate and efficient cost models?". We plan to work on more applications and different configurations to seek answers to these questions in future work.

\section{Conclusion and Future Work}

We have presented a framework, called filter-stream programming, for developing data-intensive applications in a Grid environment. This framework represents the processing in an application as a set of processing components, called filters. The goal is to constraint application components to allow for location independence, and to expose communication characteristics and resource requirements, thus enabling a runtime system to support efficient execution of the application. We have described a prototype runtime infrastructure to execute applications using the filterstream programming framework. We have discussed implementations of two data-intensive applications that make use of our filter-stream framework, and presented experimental results.

Our experimental results show that there exists a delicate balance, and sometimes subtle interactions with heterogeneous resources, that can have a large impact on application performance. We plan to further investigate such interactions to develop cost models that can aid in decomposition of applications into filters and placement of the filters. We also are in the process of implementing other applications to use the filter-stream programming framework from application areas such as volume visualization, database decision support, and image processing.

\section{References}

[1] A. Acharya, M. Uysal, and J. Saltz. Active disks: Programming model, algorithms and evaluation. In Proceedings of the Eighth International Conference on Architectural Support for Programming Languages and Operating Systems (ASPLOS VIII), pages 81-91. ACM Press, October 1998. ACM SIGPLAN Notices, Vol. 33, No. 11.

[2] A. Afework, M. D. Beynon, F. Bustamante, A. Demarzo, R. Ferreira, R. Miller, M. Silberman, J. Saltz, A. Sussman, and H. Tsang. Digital dynamic telepathology - the Virtual Microscope. In Proceedings of the 1998 AMIA Annual Fall Symposium. American Medical Informatics Association, November 1998.

[3] R. Agarwal. A super scalar sort algorithm for RISC processors. In Proceedings of 1996 ACM SIGMOD Conference, pages 240-6, 1996.

[4] Alexandria Digital Library. http://alexandria.ucsb.edu/.

[5] A. Arpaci-Dusseau, R. Arpaci-Dusseau, D. Culler, J. Hellerstein, and D. Patterson. High-performance sorting on networks of workstations. In Proceedings of 1997 ACM SIGMOD Conference, Tucson, AZ, 1997.

[6] M. D. Beynon, R. Ferreira, T. Kurc, A. Sussman, and J. Saltz. DataCutter: Middleware for filtering very large scientific 


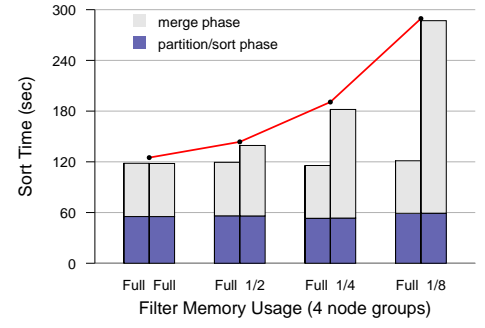

(a) Regular partitioning of input data and Partitioner filter output

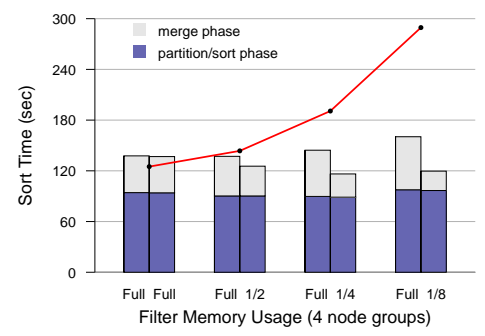

(d) Irregular partitioning of input data and Partitioner filter output (tuned)

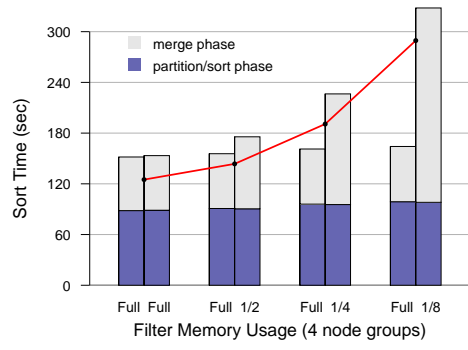

(b) Irregular partitioning of input data

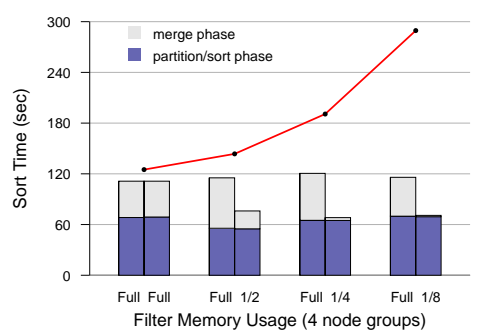

(e) Irregular partitioning of input data and Partitioner output, 2 pair of filters per Full node

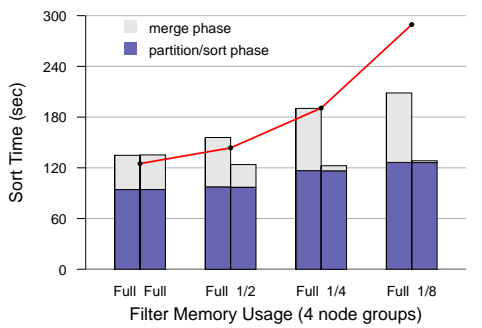

(c) Irregular partitioning of input data and Partitioner filter output

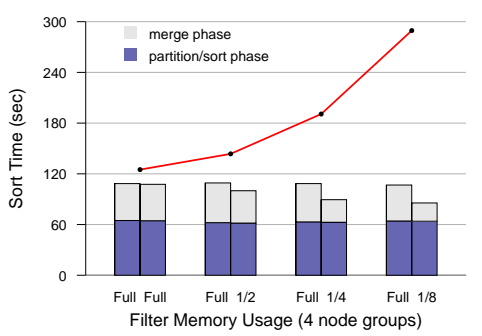

(f) Irregular partitioning of input data and Partitioner filter output (tuned), 2 pair of filters per Full node

\section{Figure 13. Execution time of sorting a 1GB dataset using 8 nodes. Two groups of four nodes: (1) memory usage held constant (Full), (2) memory usage reduced (1/X).}

datasets on archival storage systems. In Proceedings of the 2000 Mass Storage Systems Conference, College Park, MD, March 2000. IEEE Computer Society Press. To appear.

[7] A. Chervenak, I. Foster, C. Kesselman, C. Salisbury, and S. Tuecke. The Data Grid: Towards an architecture for the distributed management and analysis of large scientific datasets. (Submitted to NetStore '99), September 1999.

[8] A. Choudhary, R. Bordawekar, M. Harry, R. Krishnaiyer, R. Ponnusamy, T. Singh, and R. Thakur. PASSION: Parallel and scalable software for input-output. Technical Report SCCS-636, NPAC, September 1994. Also available as CRPC Report CRPC-TR94483.

[9] Phoenix Computers Catalog. http://www.phoenixcomputers.net/, Jan 2000. Following link from http://www.pricewatch.com/.

[10] ShopHere USA Catalog. http://205.164.161.100/products.asp?dept=33, Jan 2000. Following link from http://www.pricewatch.com/.

[11] F. Douglis and J. Ousterhout. Process migration in the Sprite operating system. In Proceedings of the Seventh International Conference on Distributed Computing Systems, pages 18-25, September 1987.
[12] I. Foster. Automatic generation of self-scheduling programs. IEEE Transactions on Parallel and Distributed Systems, 2(1):68-78, January 1991.

[13] I. Foster. Compositional parallel programming languages. ACM Trans. Prog. Lang. Syst., 18(4):454-476, July 1996.

[14] I. Foster. The Beta Grid: A national infrastructure for computer systems research. In Network Storage Symposium, October 1999.

[15] I. Foster and C. Kesselman. Globus: A metacomputing infrastructure toolkit. International Journal of Supercomputer Applications and High Performance Computing, 11(2):115128, 1997.

[16] I. Foster and C. Kesselman. The GRID: Blueprint for a New Computing Infrastructure. Morgan Kaufmann, 1999.

[17] I. Foster, C. Kesselman, and S. Tuecke. The Nexus approach to integrating multithreading and communication. Journal of Parallel and Distributed Computing, 37:70-82, 1996.

[18] I. Foster, D. Kohr, R. Krishnaiyer, and A. Choudary. Double standards: Bringing task parallelisn to HPF via the message passing interface. In Proceedings Supercomputing '96. IEEE Computer Society Press, November 1996.

[19] I. Foster, D. Kohr, R. Krishnaiyer, and J. Mogill. Remote I/O: Fast access to distant storage. In Fifth Workshop on I/O 
in Parallel and Distributed Systems (IOPADS), pages 14-25. ACM Press, 1997.

[20] Geographic Information Systems. http://www.usgs.gov/research/gis/title.html.

[21] Grid Forum. Birds-of-a-Feather Session, SC99, Nov 1999.

[22] The High Performance Storage System (HPSS). http://www.sdsc.edu/hpss/hpss 1.html.

[23] T. Johnson. An architecture for using tertiary storage in a data warehouse. In the Sixth NASA Goddard Space Flight Center Conference on Mass Storage Systems and Technologies, Fifteenth IEEE Symposium on Mass Storage Systems, 1998.

[24] W. Johnston and B. Tierney. A distributed parallel storage architecture and its potential application within EOSDIS. In the Fourth NASA Goddard Conference on Mass Storage Systems and Technologies, 1995.

[25] Land Satellite Thematic Mapper (TM). http://edcwww.cr.usgs.gov/nsdi/html/landsat_tm/ landsat_tm.

[26] K.-L. Ma and Z. Zheng. 3D visualization of unsteady 2D airplane wake vortices. In Proceedings of Visualization'94, pages 124-31, Oct 1994.

[27] Message Passing Interface Forum. Document for a standard message-passing interface. Technical Report CS-93214, University of Tennessee, November 1993.

[28] Microsoft Corp. Microsoft TerraServer. http://www.terraserver.microsoft.com, 1998.

[29] G. Patnaik, K. Kailasnath, and E. Oran. Effect of gravity on flame instabilities in premixed gases. AIAA Journal, 29(12):2141-8, Dec 1991.

[30] V. Sarkar. Partitioning and scheduling parallel programs for multiprocessors. In Research Monographs in Parallel and Distributed Computing. Cambridge, MA, 1989.

[31] SRB: The Storage Resource Broker. http://www.npaci.edu/DICE/SRB/index.html.

[32] N. Talagala, S. Asami, and D. Patterson. The Berkeley-San Francisco fine arts image database. In the Sixth NASA Goddard Space Flight Center Conference on Mass Storage Systems and Technologies, Fifteenth IEEE Symposium on Mass Storage Systems, 1998.

[33] T. Tanaka. Configurations of the solar wind flow and magnetic field around the planets with no magnetic field: calculation by a new MHD. Jounal of Geophysical Research, 98(A10):17251-62, Oct 1993.

[34] M. Teller and P. Rutherford. Petabyte file systems based on tertiary storage. In the Sixth NASA Goddard Space Flight Center Conference on Mass Storage Systems and Technologies, Fifteenth IEEE Symposium on Mass Storage Systems, 1998.

[35] M. Uysal. Programming Model, Algorithms, and Performance Evaluation of Active Disks. PhD thesis, Department of Computer Science, University of Maryland, College Park, 1999.

[36] M.-Y. Wu and D. D. Gajski. Hypertool: A programming aid for multicomputers. In Proceedings of the 1989 International Conference on Parallel Processing, pages II-15 II-18. Pennsylvania State University Press, August 1989.

[37] T. Yang and A. Gerasoulis. PYRROS: static task scheduling and code generation. In Proceedings of the 1992 International Conference on Supercomputing, July 1992. 